\title{
Financial Burden Reportedly Impact Quality of Life Among Lymphatic Filariasis Pathology Patients in Ghana
}

\section{Samuel Opoku Asiedu}

Kumasi Center for Collaborative Research

\section{Emmanuel Kobla Atsu Amewu}

Kwame Nkrumah University of Science and Technology

Priscilla Kini

Kwame Nkrumah University of Science and Technology

\section{Bill Clinton Aglomasa}

Kwame Nkrumah University of Science and Technology

\section{John Boulard Forkuor}

Kwame Nkrumah University of Science and Technology

Alexander Kwarteng ( $\nabla$ senkwarteng@yahoo.co.uk)

Kwame Nkrumah University of Science and Technology https://orcid.org/0000-0002-0893-2908

\section{Research article}

Keywords: Lymphatic Filariasis, quality of life, Ghana

Posted Date: July 13th, 2020

DOl: https://doi.org/10.21203/rs.3.rs-40332/v1

License: (1) This work is licensed under a Creative Commons Attribution 4.0 International License. Read Full License

Version of Record: A version of this preprint was published on January $21 \mathrm{st}, 2021$. See the published version at https://doi.org/10.1186/s12889-021-10170-8. 


\section{Abstract}

\section{Background}

Human lymphatic filarial pathology is the main cause of disability and poverty among people living with the infection. The second goal of the Global Programme to Eliminate Lymphatic Filariasis (GPELF) is to manage the morbidity associated with the disease with the purpose of improving the quality of life of the patients. Consequently, the current study assessed the overall quality of life of lymphatic filariasis (LF) pathology patients in some selected endemic communities in rural Ghana.

\section{Method}

In the present study, the Lymphatic Filariasis Quality of Life Questionnaires (LFSQQ) was used to evaluate the effect of lymphatic filariasis on the quality of life of people with the disease in ten (10) communities in the Ahanta West District of the Western Region of Ghana where, mass drug administration is being implemented for the past twenty years.

\section{Results}

Of the 155 study participants recruited, 115 (74.19\%) were females and 40 (25.81\%) males. A greater proportion of the study participants $(40,25.8 \%)$ were presented with stage two (2) lymphedema while only 2 patients had stage seven (7) lymphedema. The average of the overall quality of life scores of study participants was 68.24. There was a negative Pearson correlation $(r=-0.504, p$-value $<0.0001)$ between the stage of lymphedema (severity of the disease) and the quality of life of the LF patients. In addition, a clear pattern of positive correlation ( $r=0.71, p$-value $<0.001)$ was observed between the disease burden and pain/discomfort domains of the study participants. Whereas, the highest domain specific score (85.03) was observed in the domain of self-care, we noted that the environmental domain, which consist of the financial status was the lowest (45.94) among the study participants.

\section{Conclusion}

Our findings support previous works on the reduced quality of life among lymphatic filariasis patients with pathology. In this study, our results reveal a depressing financial condition among people presenting with late stages of LF pathologies, which eventually reduces their wellbeing.

\subsection{Introduction}

Lymphatic filariasis (LF) is an infection which directly impairs the lymphatics and consequently renders long-lasting disability to its victims ${ }^{1}$. LF is a mosquito-borne disease in which mosquitoes transmit the causative organisms (Wuchereria bancrofti, Brugia timori and B. malayi filarial worms) ${ }^{2}$ to uninfected persons. The disease is endemic in 83 countries with majority of the cases reported in India, one third in Africa and the remaining cases in the Pacific, the Americas, South-East Asia and the Eastern Mediterranean regions ${ }^{3}$. LF has varied form of manifestations such as hydrocele, lymphedema and 
elephantiasis ${ }^{4}$. Individuals suffering from lymphatic filariasis experience repeated filarial attacks known as adenolymphangitis (ADL), which hinders patients from actively participating in both social and economic activities ${ }^{5-7}$. Krishna et a/ 2005, identified ADL as the primary cause of disabilities among LF patients ${ }^{8}$.

In line with the strategies of the Global Programme for Elimination of Lymphatic Filariasis (GPELF) to interrupt LF transmission in endemic areas, more than 890 million individuals have participated in mass drug administration programs in 37 countries as of $2017^{4}$. Nonetheless, the tertiary component of LF elimination programme of forestalling and managing both acute and chronic disability among those already affected by disease have not achieved appreciable results. The idea of this strategy is to assist the 40 million people already affected by the disease and largely neglected, to have a better quality of life

and to be capable of engaging fully in both economic and social activities. ${ }^{4}$ The Quality of Life is defined as an individual's perception of one's position in life in the context of value systems and culture, and in relation to one's goals, standards, expectations and concerns ${ }^{9}$. Thus, it is in this perspective that WHO defines health as being "not only the absence of disease and infirmity but also the absence of physical, mental, and social well-being" ${ }^{10}$.

In order to ensure a better health-related quality of life of LF patients, the Morbidity Management and Disability Prevention (MMDP) of WHO enrolled a basic package of care. This package of care must be accessible to LF patients i.e. surgery for hydrocele, treatment for episodes of adenolymphangitis, management of lymphedema to hinder episodes of adenolymphangitis and progression of disease ${ }^{11}$. To this end, GPELF initiated the Community Home-Based Care (CHBC) concept of reaching out to individuals with varying degree of morbidities to alleviate pain and prevent disease deterioration ${ }^{3}$. Thus, the purpose of this study was to assess the Quality of life of lymphatic filariasis patients in some selected LF hotspot communities in Ghana.

\subsection{Materials And Methods}

\subsection{Study area}

The study was carried out in the Ahanta West District of Western Ghana in nine (9) communities i.e. Akatakyi, Princess Town, Cape Three Points, Asemkow, Dixcove, Ampatano, Butre, Achowa and Busua. The District is dotted with lush green hills and fertile soil with relative humidity as high as $85 \%$ in the rainy season and a little decline in humidity during the dry season. A bulk of the labour force in these villages is into activities such as fishing and farming. The study communities are lymphatic filariasis hotspots with $W$. bancrofti being the main causative organism and a microfilariae prevalence of about $20 \%{ }^{12}$.

\subsection{Study Design}


The study design was cross-sectional descriptive and conducted between March 2019 and August 2019. Persons with lymphatic filariasis were identified through communities' outreaches and individuals who were clinically diagnosed with LF and/ or manifested persistent edema of more than 6 weeks' and/or have hydrocele were potential participants for study. In addition, the potential study participants were 18 years and above and willing to consent to the study. However, individuals presenting with any form swelling or edema other than filarial-related were excluded from the study. Experienced research scientists performed the leg staging of the study participants. The WHO seven-stage system for grading of lymphedema was used as the standard for grading legs of the patients as previously described in ${ }^{5}$. The Committee of Human Research and Publications and Ethics, School of Medical Sciences, Kwame Nkrumah University of Science and Technology provided ethical clearance for this study.

\subsection{Study Instrument}

To assess the quality of life of the LF study participants, the Lymphatic Filariasis Quality of Life Questionnaires (LFSQQ) was the instrument used as described by ${ }^{13}$. The LFSQQ was administered to the study participants in their local dialects (Fante and Nzema). The instrument measured the health-related quality of LF patients through a seven-domain: mobility, self-care, daily/usual activities, disease burden, pain/discomfort, psychological health and social participation. However, for the purpose of this study, energy/fatigue, environment (which entails financial support from people in the community and safety in the neighborhood) and social relationship domains were included due to the extended family system which exist in most Ghanaian communities. Each item was scored on a 5-point scale (no problem, mild, moderate, severe, most severe). The total score was calculated based on the number of questions answered and the raw scores. Scores range from 0 to 100 with a higher score indicating a better quality of life.

\subsection{Statistical Analysis}

Data were analyzed using the Epi-Info. To do this, the overall quality of life response was calculated using the formula as described by ${ }^{14}$,

Overall QoL $=\frac{\text { Total Scores }}{\text { Higest Score (5) } \times \text { Number of Questions Anwered }} \times 100 \%$

The Domain score calculated using the formula,

Domain Score $=\frac{\text { Total score on domain }}{\text { Highest Score }(5) \times \text { Number of Questions Answered }} \times 100 \%$

To determine the relationship between the various domains of the study instruments, the Pearson Correlation and Regression analyses were performed among the various domains. The proportion of the domain scores of study participants are given in percentages and mean. 


\subsection{Result}

\subsection{Demography of Study Participants}

Taken together, 155 study participants were recruited. Three-quarters $(115,74.19 \%)$ of the study participants were females and the remaining $(40,25.81 \%)$ males. The mean age of the study participants was $52.84(S D=15.62)$. Majority of the study subjects $(97,62.58 \%)$ were involved in agrarian and/or fishing activities while occupations such as service workers, sales workers and professional related workers $(5,3.23 \% ; 12,7.74 \% ; 2,1.29 \%)$, respectively, was less common among the LF cohorts. Thirty-nine (39) of LF subjects representing $25.16 \%$ were unemployed as Table 1. A greater number of the LF patients $(84,54.19 \%)$ in this study had stage 2 lymphedema (swelling not reversible overnight). The patients with stage 1 and stage 3 were 17 (10.98\%) and 28 (18.06\%), respectively. Two (2) of the study participants with stage 7 were incapacitated and could not care for themselves whereas the remaining were with other stages as shown in Table 2.

\subsection{Quality of life Score of LFSQQ Domains among study participants}

The average overall quality of life score among the LF cohort was 68.24. Among the domains used in the study, LF pathology patients recorded the Environment domain as the lowest quality of life score (45.94), while self-care domain being the highest quality of life score (85.03). The Daily activities domain and Disease burden domain of the study instrument had a slight difference in their scores of 75.54 and 75.25, respectively. A similar trend was also observed between the Mobility domain and Pain/Discomfort domain (Figure 2).

In addition, over $80 \%$ of the study participants responded to not having "no problem with their ability to take care of themselves" (self-care) whereas over $50 \%$ of LF patients complained of severe to most severe effects of the disease on their work and also loss of strength or fatigue. In the Mobility domain, the study participants had severe problems when they sit and get out of a chair or standing for a long time. With regard to their daily activities, majority of LF patients (74\%) did not have any challenge with cooking or cleaning the floors but they had difficulties in carrying out activities such as fishing or farming. Moreover, from the responses on the Social relationship domain, averagely $58 \%$ do not have any issue interacting with people, identifying a potential spouse or having the disease affecting their relationships with their family members or neighbors. The response on the Environment domain showed that $67 \%$ of respondents indicated a little or no financial assistance when asked "how often do they get financial assistance from their relations due to their conditions"?

\subsection{Quality of life Score of LFSQQ Domains in the Community Level}


The nine (9) communities where the study was conducted had varied number of LF patients as shown in Table 1. In order to determine the overall quality score in the various LF endemic communities, a bar graph of the overall quality score among the communities was done as demonstrated in the figure 3 . The graph showed no marginal changes in the overall quality of life of LF patient residing in the communities. However, Achowa community which had only four (4) LF patients were excluded from the analysis in order give a more representative data. Moreover, considering that the Disease Burden Domain (DBD), Psychological Domain (PD) and Environment Domain (ED) were the lowest domains in the LFSQQ, a community-level distribution of these domains scores i.e. DBD, PD and ED were analyzed. As shown in (figure 4) there was general higher score in the DBD with the range (76-80) except in Dixcove where the DBD score of 66. A similar trend is also observed in ED and PD with the range of (41-53) and (56-63) respectively. However, to determine whether there was any statistical difference among the DBD, PD and ED in the communities, a one-way analysis of variance (ANOVA) was conducted, which showed no statistical difference in these domains in the community ( $p$-value 5.88). In addition, the overall quality score in the female and male was 68.42 and 67.61 respectively.

\subsection{Correlation of LF Responses on LFSSQ Domains}

To determine the relationship among the various LFSSQ domains, we performed Pearson's correlation as illustrated in Table 3. The domains of pain/discomfort and disease burden were positively correlated with a Pearson's correlation coefficient of $r=0.71$ and a $p$-value $<0.0001$ as depicted in Figure 1 . A number of the domains were moderately correlated with Pearson's correlation coefficient ( $r$ ) ranging from 0.60 0.66. The weakest correlation coefficient was observed between the domains of environment and pain/discomfort. Moreover, the linear regression model for the stage of LF (severity of the disease) and the overall quality of life score showed a negative regression with a Pearson's correlation coefficient $(r=$ -0.504 ) and $p$-value $<0.0001$ (Figure 5).

\subsection{Discussion}

This is the first study to determine the general quality of life of lymphatic filariasis patients living in Ghana even though a similar study has been conducted in India ${ }^{1}$. From our results, the overall mean QoL score for the LF subjects was 68.24, which is almost the same to the study conducted in India ${ }^{14}$ at the baseline. Nevertheless, the quality of life of LF participants in this study was slightly lower than a previous study conducted in the urban area of Tiruchirappalli in India which had an overall mean QoL score of $69.81^{1}$ using the same LFSQQ instrument. The overall quality of life index in this study indicates a good quality of life among LF patients in the coastal areas of Western Ghana, however, a higher QoL could be achieved if LF patients are taken through regular skin wash activities, exercises and other requisite hygienic practices. A six (6) months camp set up for LF patients in three endemic communities in Kerala province of India showed a significant upsurge in the QoL of the patients from 68.23 to $74.57^{14}$. 
Moreover, more than half of our LF participants $(67 \%)$ responded that they had no financial assistance from any relation. This was not surprising since the environment domain was observed to have the least domain QoL score (45.94). There are apparent reasons for such observations. First of all, the observation can be due to the dilapidating consequences of lymphatic filariasis on the patients, which rendered most of our study participants jobless or had reduced working days; making them wholly dependent on relatives and friends. This finding complements other results from this study where $29 \%$ of the respondents indicated that their condition had a negative effect on their ability to engage fully in economic activities. In addition, the stigma associated with the disease prevented some of the study participants from embarking on their jobs particularly those who were engaged in fish mongering and petty trading. The situation is further complicated as the LF usually affects poorer communities ${ }^{8,15}$ where most LF patients' mainstay financial support (relatives and friends) is most often limited. This reason is corroborated by responses given by LF infected individuals in a different study conducted in Togo ${ }^{16}$. It is worth noting from our results that $78 \%$ of the respondents felt insecure in their routine life. Although the reason for this insecurity was not immediately clear from our study, this may be due to the accustomed dejection and infamy most individual who suffer neglected tropical disease face in their respective communities $^{8}$.

A community-level analysis of the overall quality of life also revealed that the QoL score did not depend on which community the LF patients resided as it tends not to show any marginal differences across the communities of study. This may be due to the similar socio-economic status of the patients as majority of them are unemployed as an upshot of the impact of LF disease which makes them to depend on peasant farming and/or fishing activities for their livelihood. An observed general pattern of the specific domains i.e. Psychological, Disease burden and Environment domains in LFSQQ across the communities also consolidate the impression that the LF patients in this study experienced almost the same impact of the infection irrespective of the community origin. Therefore, any intervention developed to alleviate the suffering and/or to increase the quality of life of LF patients can be generalized for all and possibly replicated in other similar settings.

The severity of the disease was defined by the stage of the lymphedema. The worth of wellness of the study participants presenting the late stages of the infection was abysmal as the indicators observed declined in their overall mean QoL index. The negative Pearson's correlation $(r=-0.504, p$-value $=0.0001)$ between the severity of the disease and the quality of life of the study participant was in divergent with the positive correlation $(r=0.74, p$-value $<0.0001)$ of severity of disease and quality of life score in a different study ${ }^{1}$. Nevertheless, erstwhile study using modified Dermatology Life Quality index (mDLQI) also revealed that the severity of the filarial lymphedema had a considerable negative repercussion on the QoL of the individuals ${ }^{17}$. A possible explanation for the negative effect of the stage of the lymphedema on the quality of the respondents in our study may lie in the fact that as the swelling of their affected limbs worsened, the less productive they become and so a toiling effect on the wellbeing of LF patients. 
For further analysis on how the disease impact the physical abilities of the LF participants, we used the mobility, daily activities, pain/discomfort and self-care domains in assessing this as used elsewhere . $^{8}$ The physical ability of LF participants have been widely reported to be hampered as compared to healthy individuals without the condition ${ }^{3,4,8,11}$ and this was expected in this study. On the other hand, other studies $^{1,14}$ have also shown a higher level of self-care among LF patients which happen to corroborate with our current study where the self-care domain had the highest average QoL score of 85.03. This finding reflects the composition of our LF patients in the study where $36 \%$ of them are living with stage two (2) lymphedema and as a result could still take care of themselves.

Mobility of LF patients is also considered a key factor in assessing the physical capabilities of the patients as the various manifestations of the disease ( $A D L$, hydrocele, lymphedema and elephantiasis). This situation has a cascading effects on the employment and income status of persons living with the condition ${ }^{18,19}$. Our study participants, however, averagely had mobility domain score of 68.86 which is comparatively higher to previous studies of $43.1^{15}, 54.92$ (baseline score) ${ }^{14}$ and $52.32^{1}$. Nevertheless, this finding does not undermine the finding that $77 \%$ of the respondents had some form of difficulty in sitting and getting out of a chair or a challenge in walking a long distance. As an earlier study have also detailed the mobility of LF patients by using a $10 \mathrm{~m}$ walking test (10MWT) and a timed 'up and go' (TUG) test revealed LF patients were slower than controls $(10 \mathrm{WT}$ : cases $=0.828 \mathrm{~m} / \mathrm{s}$, controls $=1.104 \mathrm{~m} / \mathrm{s}$, TUG: cases $=14.7 \mathrm{~s}$, controls $=11.2 \mathrm{~s}$ ).

\subsection{Conclusion}

This study, carried in the LF hotspot communities in the Western Region of Ghana, shows a challenge in the quality of life of lymphatic filariasis patients as the result of the condition. Our findings also reveal financial and income difficulties among people suffering from LF, which eventually reduces their socioeconomic wellbeing. We provide compelling evidence that there is the need for relevant governmental and non-governmental stakeholders, including the Ministry for Gender and Social Protection of Ghana, to integrate some social intervention programs for people living with LF conditions in endemic regions in order to improve their wellbeing. We believe the study will inform policy about the management LF in Ghana.

\section{Declarations}

\section{Ethical Approval and Consent to participate}

The study was approved (CHRPE/AP/209/19) by the Committee of Human Research, Publications and Ethics, School of Medical Sciences, Kwame Nkrumah University of Science and Technology, KNUST, Kumasi, Ghana. Approval was also sought from Municipal Health Directorates at the Ahanta Nkwanta, Western Region, Ghana. The study protocols were explained to the participants and all participants consented to the study by signing or thumb printing the informed consent forms 


\section{Consent for Publication}

Not Applicable

\section{Availability of data and materials}

Not Applicable

\section{Competing Interest}

The authors declare no conflict of interest.

\section{Authors' contributions}

AK conceived the idea, SOA collected the data from the field and conducted the statistical analyses and drafted the manuscript, EKAA, PK and BCA assisted in the field work and edited paper. JBF also read the manuscript critically and equaled edited it. All authors approved the final version of this article

\section{Acknowledgements}

The authors would like to thank Mr. Samuel Dodge, The Disease Control Officer at the Ahanta West Health Directorate for his immense contribution to this study.

\section{Authors' Details}

${ }^{1}$ Kumasi Centre for Collaborative Research in Tropical Medicine, Kwame Nkrumah University of Science and Technology, Kumasi-Ghana

${ }^{2}$ Department of Biochemistry and Biotechnology, Kwame Nkrumah University of Science and Technology, Kumasi, Ghana

${ }^{3}$ Department of Sociology and Social Work, Kwame Nkrumah University of Science and Technology, Kumasi, Ghana

\section{Reference}

1. Hemalatha, K. \& V, R. P. Impact of Lymphatic Filariasis on Quality of Life of Affected Individuals: A Community Based Cross Sectional Survey. 6, 13-18 (2016).

2. Streit, T. \& Lafontant, J. G. Eliminating lymphatic filariasis: A view from the field. Ann. N. Y. Acad. Sci.1136, 53-63 (2008).

3. World Health Organization (WHO). Weekly epidemiological record. (2004).

4. Programme, T. G., Filariasis, E. L. \& Programme, L. Weekly epidemiological record Relevé épidémiologique hebdomadaire. 589-604 (2018). 
5. Kwarteng, A. et al. Influence of seasonal variation on reported filarial attacks among people living with lymphedema in Ghana. 1-7 (2019). doi:10.1186/s12879-019-4084-2

6. McPherson, T. et al. Interdigital lesions and frequency of acute dermatolymphangioadenitis in lymphoedema in a filariasis-endemic area. Br. J. Dermatol.154, 933-941 (2006).

7. Dai, M. et al. Dermal Structure in Lymphedema Patients with History of Acute Dermatolymphangioadenitis Evaluated by Histogram Analysis of Ultrasonography Findings: A CaseControl Study. Lymphat. Res. Biol.14, 2-7 (2016).

8. Kumari, A. K., Harichandrakumar, K. T., Das, L. K. \& Krishnamoorthy, K. Physical and psychosocial burden due to lymphatic filariasis as perceived by patients and medical experts. Trop. Med. Int. Heal.10, 567-573 (2005).

9. Bernstein, U. Study of behaviour in wear and wear life of shirts-an example from practical textile testing. 41, (1972).

10. WHO. WHO definition of Health,Preamble to the Constitutionnof the World Health Organization. in International Health Conference NY19-22 (1946).

11. WHO. Lymphatic Filariasis: Managing Morbidity and Preventing Disability World Health Organization an Aide-Mémoire for National Programme Managers. (2013).

12. Dunyo, S. K. et al. Lymphatic filariasis on the coast of Ghana. Trans. R. Soc. Trop. Med. Hyg.90, 634638 (1996).

13. Thomas, C. et al. Comparison of Three Quality of Life Instruments in Lymphatic Filariasis: DLQI, WHODAS 2.0, and LFSQQ. PLoS Negl. Trop. Dis.8, 19-21 (2014).

14. Aggithaya, M. G. et al. Self care integrative treatment demonstrated in rural community setting improves health related quality of life of lymphatic filariasis patients in endemic villages. Acta Trop.126, 198-204 (2013).

15. Stanton, M. C. et al. Measuring the physical and economic impact of filarial lymphoedema in Chikwawa district, Malawi: A case-control study. Infect. Dis. Poverty6, 1-9 (2017).

16. Richard, S. A., Mathieu, E., Addiss, D. G. \& Sodahlon, Y. K. A survey of treatment practices and burden of lymphoedema in Togo. Trans. R. Soc. Trop. Med. Hyg.101, 391-397 (2007).

17. Chandrasena, T. G. A. N., Premaratna, R., Muthugala, M. A. R. V, Pathmeswaran, A. \& de Silva, N. R. Modified Dermatology Life Quality Index as a measure of quality of life in patients with filarial lymphoedema. Trans. R. Soc. Trop. Med. Hyg.101, 245-249 (2007).

18. Coreil, J., Mayard, G., Louis-Charles, J. \& Addiss, D. Filarial elephantiasis among Haitian women: Social context and behavioural factors in treatment. Trop. Med. Int. Heal.3, 467-473 (1998).

19. Perera, M., Whitehead, M., Molyneux, D., Weerasooriya, M. \& Gunatilleke, G. Neglected patients with a neglected disease? A qualitative study of lymphatic filariasis. PLoS Negl. Trop. Dis.1, (2007).

\section{Tables}

Table 1: Demography of Study Participants 


\begin{tabular}{|c|c|}
\hline SEX & $\mathrm{n}(\%)^{\mathrm{a}}$ \\
\hline Female & $115(74.19)$ \\
\hline Male & $40(25.81)$ \\
\hline \multicolumn{2}{|l|}{ AGE } \\
\hline$(\operatorname{mean} \pm \mathrm{SD})$ & $52.84 \pm 15.6295 \% \mathrm{CI}(50.34-55.33)$ \\
\hline \multicolumn{2}{|l|}{ Occupation } \\
\hline Agricultural/Fishman/Fishmonger/Farmer & $97(62.58)$ \\
\hline Service workers & $5(3.23)$ \\
\hline Sales workers & $12(7.74)$ \\
\hline Professionals/Pensioner/teacher & $2(1.29)$ \\
\hline Unemployed & $39(25.16)$ \\
\hline \multicolumn{2}{|l|}{ Community } \\
\hline Achowa & $4(2.58)$ \\
\hline Akatakyi & 37 (23.87) \\
\hline Ampatano & $21(13.55)$ \\
\hline Asemkow & $24(15.48)$ \\
\hline Busua & $13(8.39)$ \\
\hline Butre & $14(9.03)$ \\
\hline Cape 3 points & $10(6.45)$ \\
\hline Dixcove & $16(10.32)$ \\
\hline Princess Town & $16(10.32)$ \\
\hline
\end{tabular}

This table shows the various sections of the demography of the study participants with CI as Confidence interval, aPercentage (\%) is the number (n) divided by 155 (total N).

Table 2: Clinical profile of the patients

\begin{tabular}{|l|ll|}
\hline \multicolumn{2}{|c|}{ Stages of Lymphoedema } & \multicolumn{1}{c|}{$\mathrm{n}(\%)^{\mathrm{a}}$} \\
\hline 1 & Swelling reversible overnight & $17(10.98)$ \\
2 & Swelling not reversible overnight & $84(54.19)$ \\
3 & Shallow skin folds & $28(18.06)$ \\
4 & Skin knobs & $6(3.87)$ \\
5 & Deep skin folds & $10(6.45)$ \\
6 & Presence of “mossy lesions" & $8(5.16)$ \\
7 & Unable to care for self & $2(1.29)$ \\
\hline
\end{tabular}


This table depicts the staging of the Lymphedema of the study participants, aPercentage (\%) is the number ( $\mathrm{n}$ ) divided by 155 (total N).

Table 3. Pearson's Correlation for the Domains of Quality of Life Scores LF Patients.

\begin{tabular}{|c|c|c|c|c|c|c|c|c|c|}
\hline Variables & 1 & 2 & 3 & 4 & 5 & 6 & 7 & 8 & 9 \\
\hline 1. Mobility & - & & & & & & & & \\
\hline 2. Daily Activities & $0.62 * *$ & - & & & & & & & \\
\hline 3. Self-care & $0.65 * *$ & $0.60 * *$ & - & & & & & & \\
\hline 4. Disease/ Burden & $0.65 * *$ & $0.56^{*}$ & $0.58 *$ & - & & & & & \\
\hline 5. Pain/Discomfort & $0.66 * *$ & $0.50 *$ & $0.56 *$ & $0.71 * * *$ & - & & & & \\
\hline 6. Work/Fatigue & $0.63 * *$ & $0.62 * *$ & $0.64 * *$ & $0.63 * *$ & $0.58 *$ & - & & & \\
\hline 7. Psychological Health & 0.43 & 0.41 & $0.50 *$ & 0.40 & 0.45 & 0.48 & - & & \\
\hline 8. Social Relationships & 0.49 & $0.53 *$ & $0.54 *$ & $0.53 *$ & 0.46 & $0.56^{*}$ & $0.53 *$ & - & \\
\hline 9. Environment & 0.24 & 0.32 & 0.23 & 0.16 & 0.09 & 0.23 & 0.47 & $0.51 *$ & - \\
\hline
\end{tabular}

***Strong Correlation, ** Moderate Correlation and *Weak Correlation.

\section{Figures}




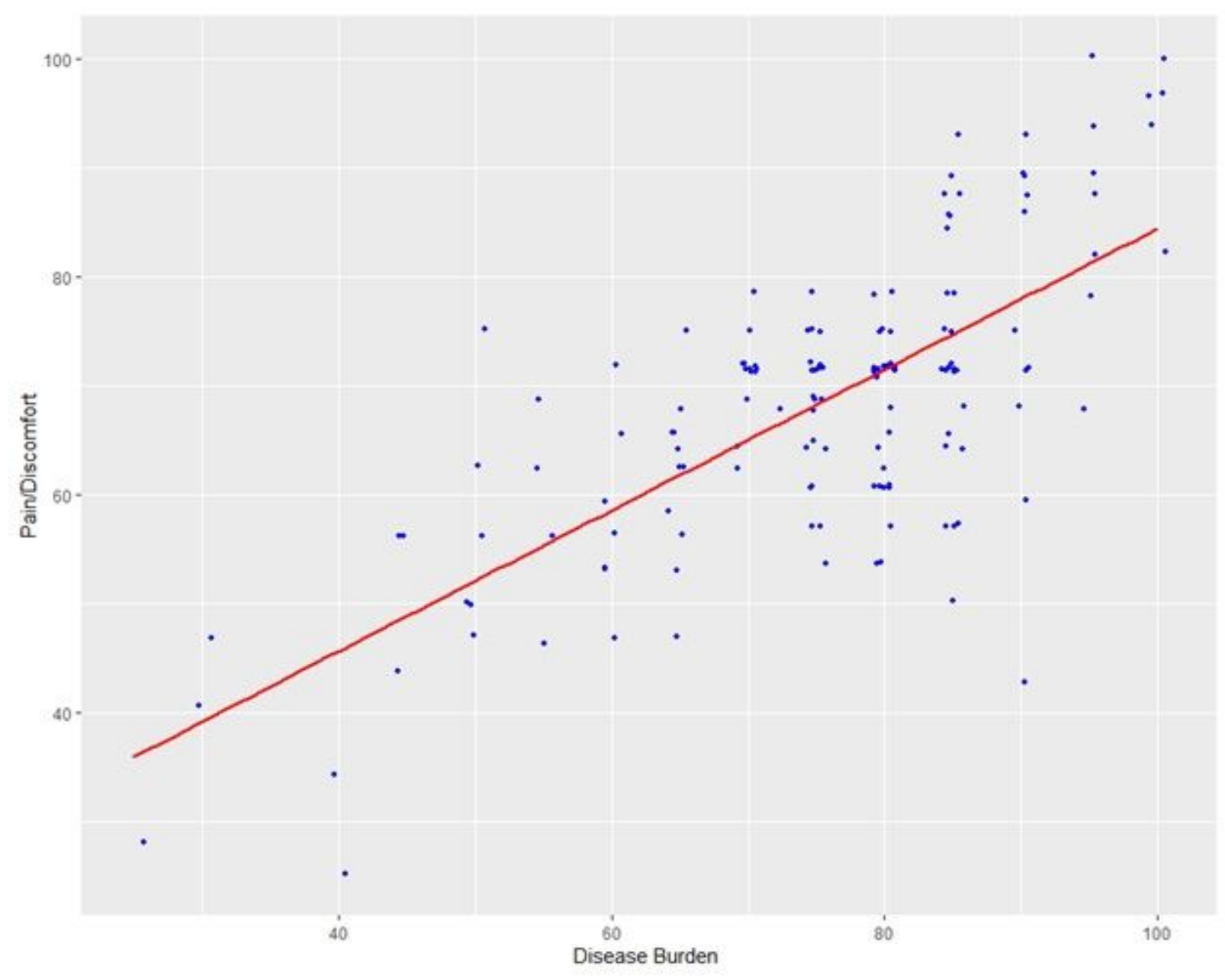

Figure 1

The Correlation result between the Disease Burden and Pain/Discomfort Domains 


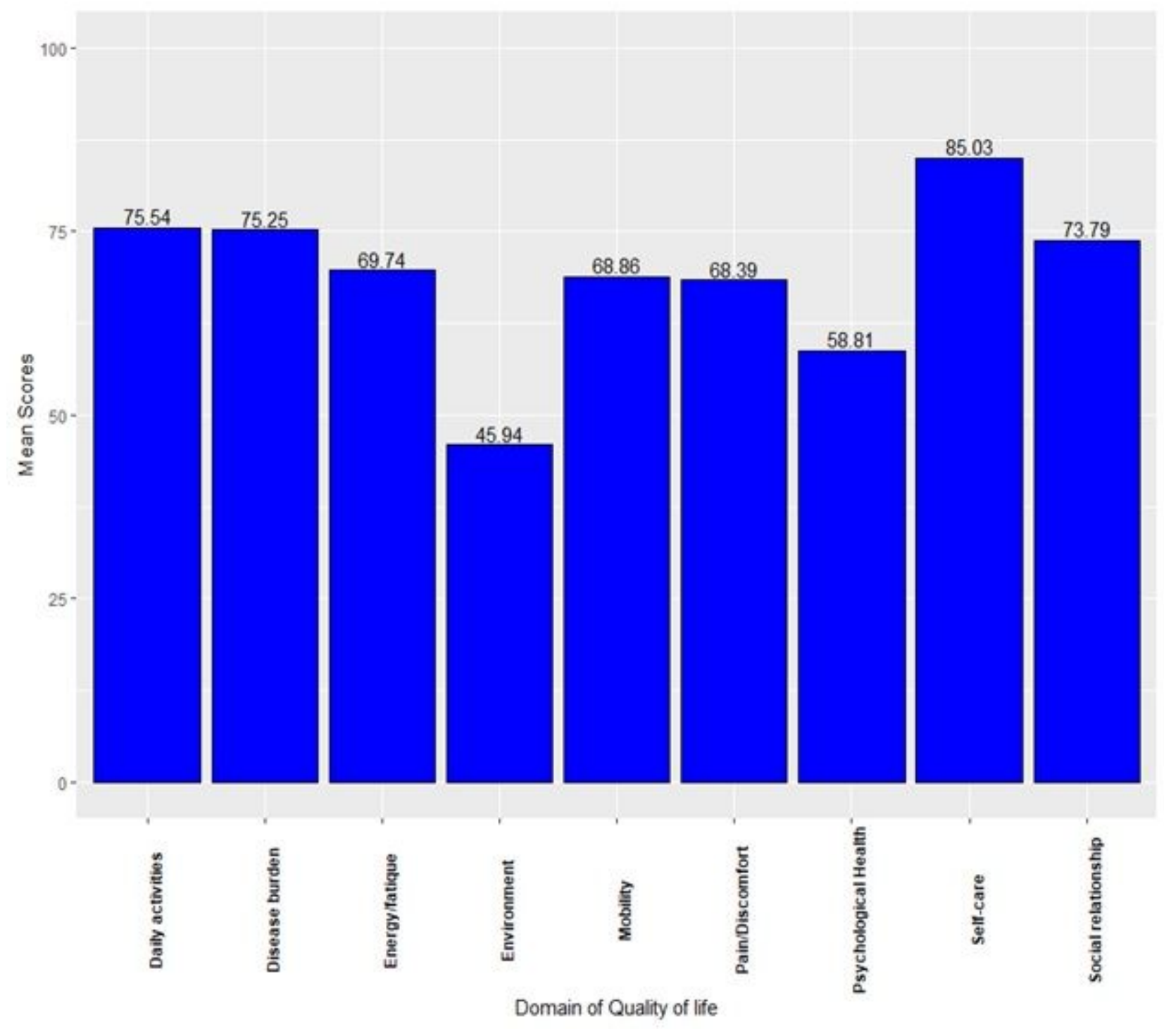

Figure 2

Bar Chart depicting the mean quality life scores of LF patients for each Domain. 


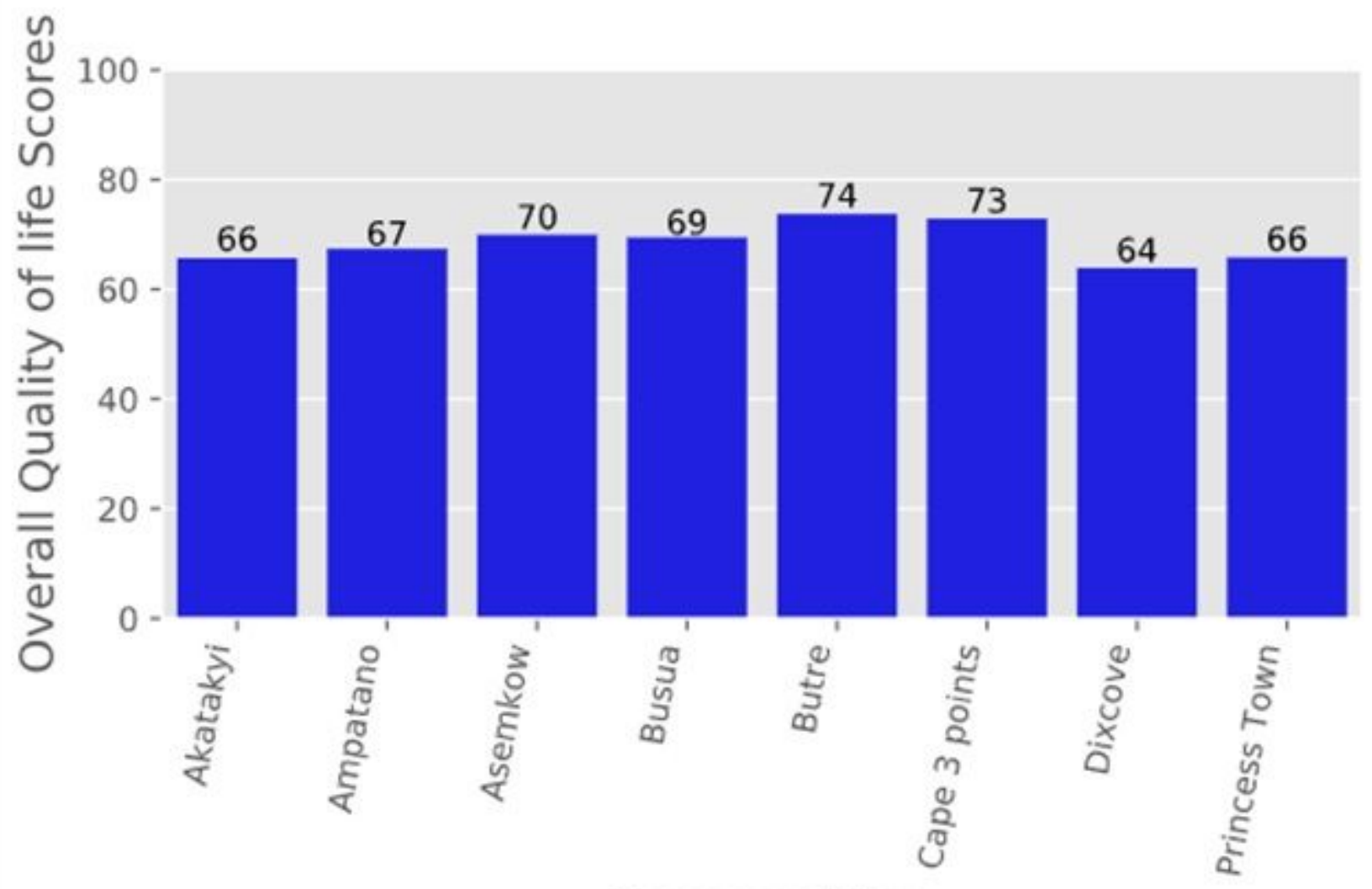

Communities

Figure 3

The Overall Quality Life Scores in the Communities 


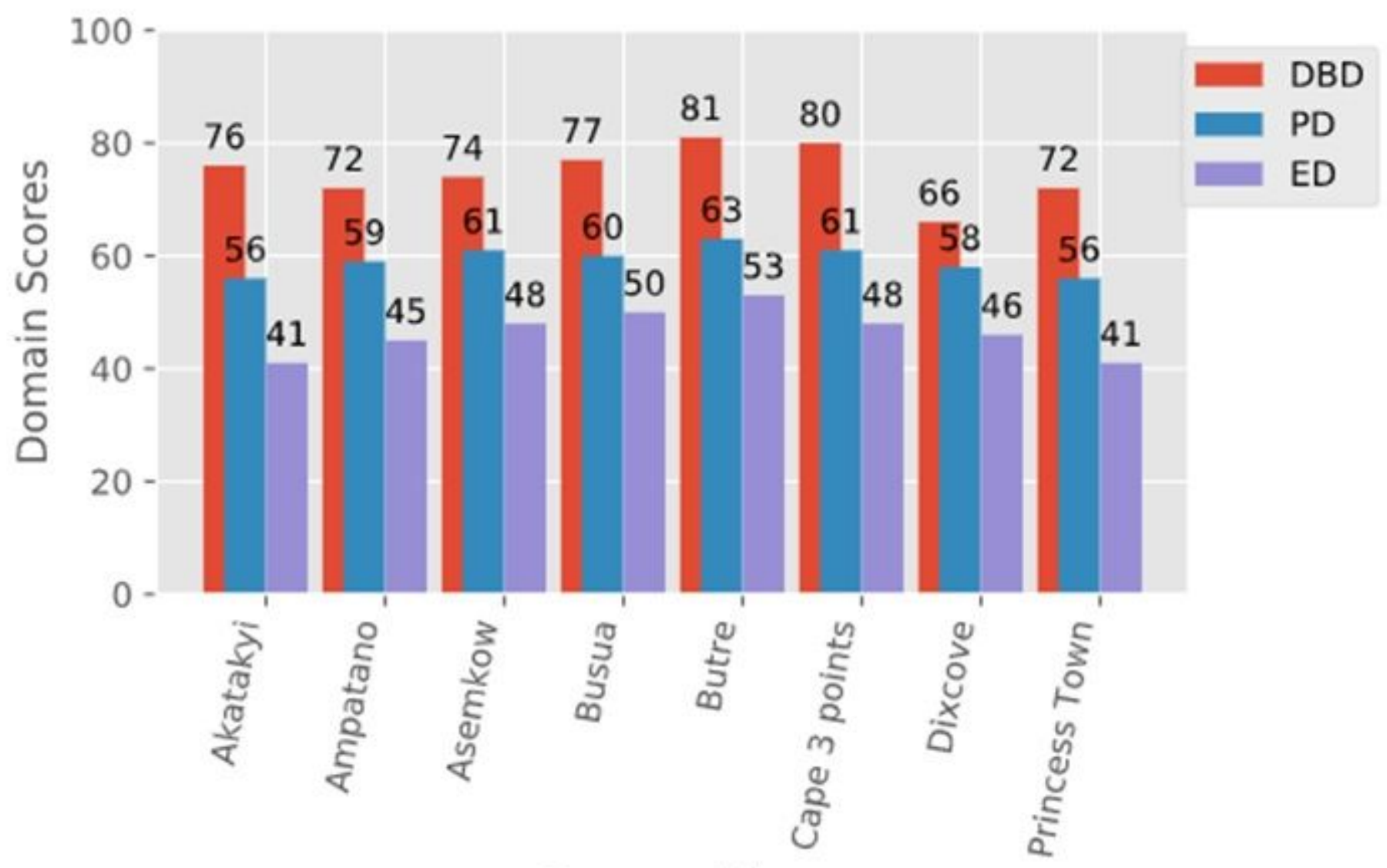

Communities

\section{Figure 4}

The Distribution of Domains Scores in the Community This graph shows the domains scores of DBD, PD and ED in the community of study *DBD: Disease Burden Disease, * PD: Psychological Domain, *ED: Environment Domain. 


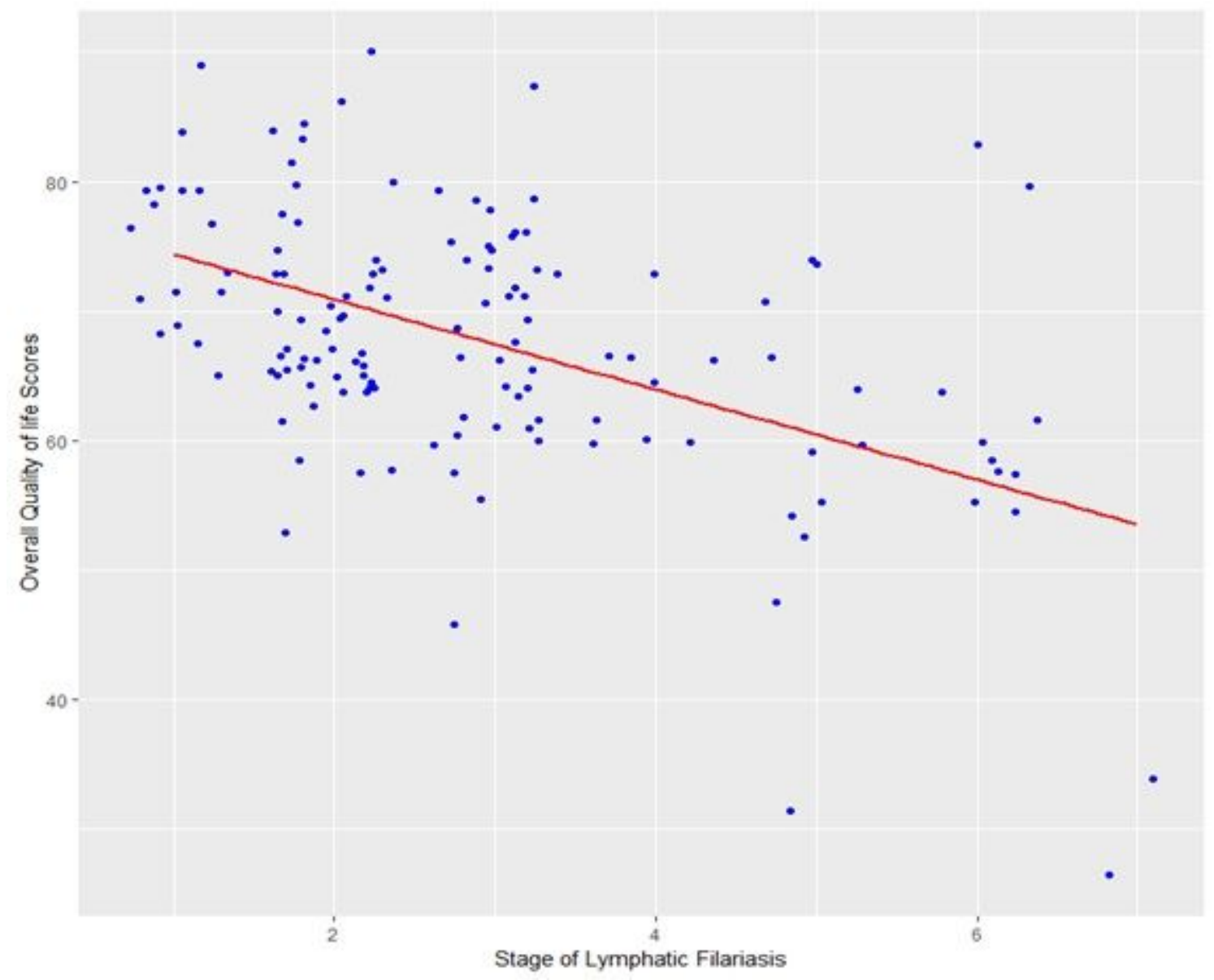

Figure 5

The correlation result of the severity of disease and overall Quality of Life Scores 